\title{
IMAGE RESTORATION BASED ON CONSTRAINED TOTAL LEAST SQUARES
}

\author{
Xiangchao Gan ${ }^{1}$, Alan Wee-Chung Liew ${ }^{1}$ and Hong Yan ${ }^{1,2}$ Senior Member IEEE \\ ${ }^{1}$ Department of Computer Engineering and Information Technology \\ City University of Hong Kong, 83 Tat Chee Avenue, Kowloon, Hong Kong \\ ${ }^{2}$ School of Electrical and Information Engineering \\ University of Sydney, NSW 2006, Australia
}

\begin{abstract}
In constrained total least squares algorithm (CTLS), the selection of a minimal algebraic set of linearly independent random variables to express the noise matrix $\Delta C$ is an important task. In this paper, A fast algorithm is provided using the possibly dependent random variables set. We showed that it can be viewed as a combination of Mesarovic et al's CTLS method and RLS method when the noise is Gaussian. Our experimental study indicated that our algorithm has better visual and objective quality, while having a much lower computation cost. Moreover, our algorithm can also handle a more general noise model.
\end{abstract}

\section{INTRODUCTION}

The Total Least Squares (TLS) method has been widely used in image restoration. TLS solution is obtain by

$$
\begin{gathered}
\min _{\mathbf{x}, \Delta A, \Delta \mathbf{b}}\|[\Delta A: \Delta \mathbf{b}]\|_{\text {Frob }} \\
\text { subject to } \mathbf{b}+? \mathbf{b}=(A+\Delta A) \mathbf{x}
\end{gathered}
$$

where $\mathbf{b}, \mathbf{x}, \Delta \mathbf{b} \in R^{L}$ represent the observed degraded image, the original image, and the additive noise, respectively. $A$ is the known PSF and $\Delta A$ is zero mean random noise matrix.

In a deconvolution problem, the matrix $A$ is circulant, and the TLS method does not preserve the circulant structure of $A$. Let $C=[A \vdots \mathbf{b}]$ and $\Delta C=[\Delta A \vdots ? \mathbf{b}]$. Under this circumstance, one choice is to formulate the problem as

$$
\begin{aligned}
& \min _{\Delta C, \mathbf{x}}\|\Delta C\|_{\text {Frob }}^{2} \quad \text { subject to } \quad(C+\Delta C)\left(\begin{array}{c}
\mathbf{x} \\
-1
\end{array}\right)=0, \\
& \text { subject to } \quad \Delta C \text { having the same structure as } C .
\end{aligned}
$$

In [1], Abatzoglou proposed a Constraint Total Least Squares (CTLS) method,

$$
\begin{gathered}
\min _{\mathbf{v}, \mathbf{x}}\|\mathbf{G}\|_{Q}^{2} \quad \text { subject to }(C+\Delta C)\left(\begin{array}{c}
\mathbf{x} \\
-1
\end{array}\right)=0 \\
\text { subject to } \Delta C=\left[F_{1} \mathbf{v} \vdots F_{2} \mathbf{v} \vdots \ldots \vdots F_{L+1} \mathbf{v}\right]
\end{gathered}
$$

The matrix $\mathrm{Q}$ is related to the known noise property. a way is provided to avoid computing it directly. The procedure involves first finding a minimal algebraic set of linearly independent random variables from $\Delta C$ to form $\mathbf{v}$. The noise property of $\mathbf{v}$ is assumed known, i.e., its correlation matrix $R=E\left\{\mathbf{v} \mathbf{v}^{T}\right\}=P P^{T}$ is known. Then,

performs a whitening of $\mathbf{v}$ to get $\mathbf{u}=P^{-1} \mathbf{v}$. Equation (2) can then be written as

$$
\min _{\mathbf{u}, \mathbf{x}}\|\mathbf{u}\|^{2}
$$

$$
\text { subject to }\left(C+\left[F_{0} P \mathbf{u}: F_{1} P \mathbf{u}: \cdots \vdots F_{L+1} P \mathbf{u}\right]\right)\left(\begin{array}{c}
\mathbf{x} \\
-1
\end{array}\right)=0
$$

which is a quadratic minimization problem subjected to a constraint. Finally, equation (B) is transformed into an unconstrained minimization problem as shown below (see theorem 1 in [1]),

$$
\begin{gathered}
\min _{x}\{P(\mathbf{x})\} \\
\text { where } P(\mathbf{x})=(A \mathbf{x}-\mathbf{b})^{t}\left(H_{x}^{\dagger}\right)^{t} H_{x}^{\dagger}(A \mathbf{x}-\mathbf{b})
\end{gathered}
$$

and $H_{x}=\sum_{i=1}^{L} x_{i} F_{i} P-F_{L+1} P$.

\section{CTLS MTHOD USING DEPENDENT RANDOM VARIABLES SET}

$$
\begin{aligned}
& \text { Let the CTLS restoration be modeled as } \\
& \begin{aligned}
\mathbf{b}+\mathbf{?} \mathbf{b}=(A+\Delta A) \mathbf{x}, \text { if let } \\
\qquad \mathbf{r}^{\prime}=[\Delta A(0,0), \Delta A(1,0) \cdots, \Delta A(L-1,0)]^{t} \\
W_{1}^{\prime}=E\left\{? \mathbf{r}^{\prime} ? \mathbf{r}^{\prime *}\right\} \\
\mathbf{?} \mathbf{b}=[\Delta b(0), \cdots \Delta b(L-1)]^{t} \\
W_{2}=E\left\{? \mathbf{b} ? \mathbf{b}^{*}\right\}=G G^{*}
\end{aligned}
\end{aligned}
$$




$$
\mathbf{u}=P^{\dagger} \mathbf{v}=\left[\begin{array}{c}
R^{\dagger} \boldsymbol{?} \mathbf{r} \\
\cdots \cdots \\
G^{-1} \boldsymbol{?} \mathbf{b}
\end{array}\right]
$$

In [5], we prove that this method can provide a good solution for CTLS problem. Then equation (3)

$$
\min _{\mathbf{u}, \mathbf{x}}\|\mathbf{u}\|^{2}
$$

subject to $A \mathbf{x}-\mathbf{b}+H_{x} \mathbf{u}=0$

where

$$
Z=\left[\begin{array}{ccccc}
x(0) & x(L-1) & \cdots & \cdots & x(1) \\
x(1) & x(0) & & & x(2) \\
x(2) & x(1) & \ddots & & x(3) \\
\vdots & \vdots & & \ddots & \vdots \\
x(L-1) & x(L-2) & \cdots & \cdots & x(0)
\end{array}\right]
$$

If $W_{1}$ and $W_{2}$ are circulant. In DCT transform domain, equation (27) is equivalent to [2]

$$
\begin{gathered}
\min _{X(u, v)}\{P(X(u, v))\} \\
P(X(u, v))=\frac{|A(u, v) X(u, v)-B(u, v)|^{2}}{S_{\Delta v}(u, v)|X(u, v)|^{2}+S_{\Delta b}(u, v)} \\
u=0,1, \cdots, M-1 \text { and } v=0,1, \cdots, N-1
\end{gathered}
$$

$X(u, v)$ and $B(u, v)$ are the 2-D DFT of the corresponding spatial-domain images. $A(u, v)$ is the eigenvalues of the circulant matrix $A$, and can be replaced by the Optical Transfer Function (OTF) The detail analysis of $S_{\Delta r}(u, v)$ and $S_{\Delta b}(u, v)$ are given later.

To get a solution with good visual effect, smoothness constraint is needed. As in [1], we also use regularization to constrain our algorithm. The regularized version of (7) is

$$
\min _{X(u, v)}\{P(X(u, v))\},
$$

where

$$
\begin{aligned}
& P(X(u, v))=\frac{|A(u, v) X(u, v)-B(u, v)|^{2}}{S_{\Delta r}(u, v)|X(u, v)|^{2}+S_{\Delta b}(u, v)}+\lambda|Q(u, v)|^{2}|X(u, v)|^{2} \\
& u=0,1, \cdots, M-1 \text { and } v=0,1, \cdots, N-1
\end{aligned}
$$

\section{3. . A FAST METHOD TO GET THE SOLUTION OF CTLS EQUATION}

We assume that the noise in PSF and $\boldsymbol{b}$ are Gaussian and white, with variance denoted by $\sigma_{P S F}^{2}$ and $\sigma_{\Delta \mathbf{b}}^{2}$, respectively. The circular convolution is adopted at the boundary of the image in the degradation model. The PSF and the image $f(m, n)$ have $L_{1} \times L_{2}$ and $M \times N$ components, respectively. According to Lemma 2, we obtain,

$$
W_{1}=\left[\begin{array}{ccccc}
W^{1} & 0 & 0 & \cdots & 0 \\
0 & W^{2} & 0 & \cdots & \vdots \\
0 & 0 & \ddots & 0 & \vdots \\
\vdots & \vdots & 0 & \ddots & 0 \\
0 & \cdots & \cdots & \cdots & W^{N}
\end{array}\right]
$$

where

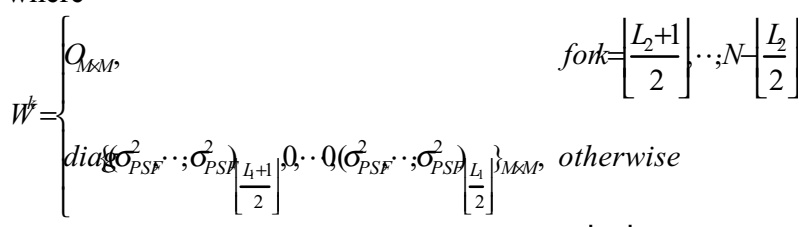

and $O_{M \times M}$ are $M \times M$ zero matrices, and $\lfloor c\rfloor$ is used to denote the greatest integer not exceeding $c$.

In our experiment, we find that letting $S_{\Delta r} \approx W_{1}$ is a good choice

$S_{\Delta r}(u, v)= \begin{cases}\sigma_{P S F}^{2}, & \left.\text { for } u=-\left\lfloor\frac{L_{1}}{2}\right\rfloor, \cdots, \mid \frac{L_{1}-1}{2}\right\rfloor \text { and } v=-\left\lfloor\frac{L_{2}}{2}\right\rfloor, \cdots,\left\lfloor\frac{L_{2}-1}{2}\right\rfloor \\ 0, & \text { otherwise }\end{cases}$ Then, equation (8) can be separated into two parts,

$$
\begin{aligned}
& X(u, v)= \begin{cases}\min _{X u, v}(P(X(u, v))\}, & \text { for } \left.u=\left\lfloor-\frac{L_{1}}{2}|, \cdots ;| \frac{L_{1}-1}{2}\right\rfloor \text { and } v=-\left\lfloor\frac{L_{2}}{2}\right\rfloor \ldots, \mid \frac{L_{2}-1}{2}\right\rfloor \\
\frac{A(u, v) B(u, v)}{\left.\left|A(u, v)^{2}+\lambda\right| Q(u, v)\right|^{2} \sigma_{\Delta}^{2}}, & \text { otherwise }\end{cases} \\
& P(X(u, v))=\frac{|A(u, v) X(u, v)-B(u, v)|^{2}}{\sigma_{P S F}^{2}|X(u, v)|^{2}+\sigma_{\Delta \mathrm{b}}^{2}}+\lambda|Q(u, v)|^{2}|X(u, v)|
\end{aligned}
$$

Using our dependent set for $\mathbf{v}$, we found that Mesarovic et al's method can be regarded as an approximation of equation (6), i.e., if we replace all the zeros along the diagonal direction with $\sigma_{P S F}^{2}$, then $W_{1}$ becomes a circulant matrix,

$$
\begin{gathered}
\quad W_{1}^{\prime}=\left[\begin{array}{ccccc}
\sigma_{P S F}^{2} & 0 & 0 & \cdots & 0 \\
0 & \sigma_{P S F}^{2} & 0 & \cdots & \vdots \\
0 & 0 & \ddots & 0 & \vdots \\
\vdots & \vdots & 0 & \ddots & 0 \\
0 & \cdots & \cdots & \cdots & \sigma_{P S F}^{2}
\end{array}\right]_{M N \times M N} \\
S_{\Delta r}^{\prime}(u, v)=\sigma_{P S F}^{2}, \\
\text { for } u=0,1, \cdots, M-1 \text { and } v=0,1, \cdots, N-1
\end{gathered}
$$


and we obtain the solution of Mesarovic et al's method as shown below,

$$
\begin{gathered}
\min _{X(u, v)}\{P(X(u, v))\} \\
P(X(u, v))=\frac{|A(u, v) X(u, v)-B(u, v)|^{2}}{\sigma_{P S F}^{2}|X(u, v)|^{2}+\sigma_{\Delta \mathbf{b}}^{2}}+\lambda|Q(u, v)|^{2}|X(u, v)| \\
u=0,1, \cdots, M-1 \text { and } v=0,1, \cdots, N-1
\end{gathered}
$$

However, this approximation is far from being the best one, since the total power of the noise spectrum $S^{\prime}{ }_{\Delta r}(u, v)$ is several times greater than that before approximation when the size of the PSF is much less than that of the image. Thus, the noise power is amplified several times in the solution. We can prove that equation (9) is a much better approximation of $S_{\Delta_{r}}(u, v)$ in Frobenius norm.

Proof: We need to prove that

$$
\left\|W W_{1} W^{H}-W_{1}^{\prime}\right\|_{\text {Frob }} \geq\left\|W W_{1} W^{H}-W_{1}\right\|_{\text {Frob }}
$$

Since $W_{1}$ and $W_{1}^{\prime}$ are both diagonal, we have

$$
\begin{gathered}
\left(W W_{1} W^{H}-W_{1}^{\prime}\right)_{(i, j)}=\left(W W_{1} W^{H}-W_{1}\right)_{(i, j)} \\
\text { for } i \neq j
\end{gathered}
$$

And since $W_{1}$ is diagonal, we have

$$
\begin{aligned}
\left(W W_{1}\right)_{(i, j)}= & W_{(i, j)} W_{1(j, j)} \\
\left(W W_{1} W^{H}\right)_{(i, i)} & =\sum_{u=0}^{M N-1} W_{(i, u)} W_{1(u, u)} W_{(u, i)}^{H} \\
& =\sum_{u=0}^{M N-1} W_{1(u, u)}=\frac{L_{1} L_{2}}{M N} \sigma_{P S F}^{2}
\end{aligned}
$$

Then,

$$
\begin{gathered}
\sum_{i=0}^{M N-1}\left(W W_{1} W^{H}-W_{1}^{\prime}\right)_{(i, i)}{ }^{2}-\sum_{i=0}^{M N-1}\left(W W_{1} W^{H}-W_{1}\right)_{(i, i)}{ }^{2} \\
=\left(1-\frac{L_{1} L_{2}}{M N}\right)^{2} M N \sigma_{P S F}^{4}-\left(1-\frac{L_{1} L_{2}}{M N}\right)^{2} L_{1} L_{2} \sigma_{P S F}^{4} \\
\quad-\left(\frac{L_{1} L_{2}}{M N}\right)^{2}\left(M N-L_{1} L_{2}\right) \sigma_{P S F}^{4} \\
=\left(1-\frac{2 L_{1} L_{2}}{M N}\right)\left(M N-L_{1} L_{2}\right) \sigma_{P S F}^{4}
\end{gathered}
$$

In image processing, the PSF size is often much less than the size of the image, that is $1-\frac{2 L_{1} L_{2}}{M N}>0$, so

$$
\left\|W W_{1} W^{H}-W_{1}^{\prime}\right\|_{F r o b} \geq\left\|W W_{1} W^{H}-W_{1}\right\|_{F r o b}
$$

The equation of (10) differs from ((13) in that the former uses $\sigma_{P S F}^{2}=0$ for high frequencies while the latter estimates the entire DFT coefficients. When the variance of the noise in the PSF, that is $\sigma_{P S F}^{2}$, becomes zero, the regularized CTLS estimate degenerates to the RLS estimate [4]. Our algorithm uses the CTLS formulation only for the low frequencies. For the high frequencies, our algorithm uses the RLS estimate. From this viewpoint, our algorithm can be seen as a combination of the TLS and RLS methods.

In [1], the Davidon-Fletcher-Powell optimization technique is used to find each of the optimal value $X(u, v)$ for every $\{u, v\}$. In our algorithm, the optimization only needs to be applied to the low frequency part, thus resulting in great computational saving.

\section{NUMERICAL EXPERIMENTS}

In this section, we present numerical experiments to verify our algorithm. Due to the similarity of our algorithm with that of [2] [3], their choice of the $\lambda$ parameter and can be modified to fit our algorithm. The perturbation analysis of the CTLS algorithm in [2] is applicable for our algorithm.

For comparison, we use the experimental data of [2] in our experiments. The $256 \times 256$ "LENA" image is used as the source image. The Gaussian PSF used to blur the source image has a variance of $\sigma^{2}=6.25$, and the region of support is $29 \times 29$, The PSF was corrupted with additive white Gaussian noise of variance $\sigma_{\Delta A}^{2}=8 \cdot 10^{-7}$. Gaussian noise with $\sigma_{\Delta b}^{2}=1.0$ was used for ? b .

1000 Monte Carlo simulations were performed with independent realizations of the stochastic blur and the noise. As a quantitative measure of performance, the improvement in signal-to-noise-ratio (ISNR) was used [2],

$$
I S N R=20 \log \frac{\|f-g\|_{2}}{\|f-\hat{f}\|_{2}}
$$

where $f, g$, and $\hat{f}$ are the original, degraded, and estimated images, respectively.

The quantitative results are given in Table 1 and the visual results are given in Fig.1. From the experiments, we observe that our algorithm has better visual and objective quality. 


\begin{tabular}{|c|c|c|c|c|}
\hline & \multicolumn{4}{|c|}{ ISNR (db) } \\
\hline$\sigma_{\Delta A}^{2} \quad \sigma_{\Delta b}^{2}$ & LMMSE & RLS & Mesarovic's & Our's \\
\hline $8 \cdot 10^{-7} 1.0$ & -0.77 & -2.12 & 1.17 & 4.89 \\
\hline
\end{tabular}

Table 1 ISNR comparison for four methods

\section{CONCLUSION}

In this paper, we use the constrained total least-square (CTLS) method to solve the image restoration problem. In conventional CTLS algorithm, the selection of a minimal algebraic set of linearly independent random variables to express the noise matrix $\Delta C$ is an important requirement. This makes the CTLS equation very sensitive to the size of the PSF, and limits the application of the CTLS method. In this paper, a possibly dependent set could also be used without affecting the final result. We showed that, by using a specially selected set and the property of DFT, our CTLS equation can be decoupled into a set of much simpler equations, which makes the computation substantially more efficient. When the noise is Gaussian, our algorithm can be viewed as a combination of Mesarovic et al's method and the RLS method. Moreover, our algorithm can also handle a more general noise model. Simulation experiments indicated that our algorithm has better visual and objective quality, but with much lower computation.

Finally, we like to remark that the sparseness structure is not only applicable for the situation where the PSF size is smaller than that of the image, but also for the situation where part of the PSF values are known or fixed.

\section{REFERENCES}

[1]. T. J. Abatzoglou, J. M. Mendel, and G. A. Harada, "The constrained Total Least Squares Technique and its Applications to Harmonic Superesolution", IEEE Trans. Signal Processing, vol. 39, pp. 1070-1087, May 1991

[2]. V. Z. Mesarovic, N. P. Galatsanos, and A. K. Katsaggelos, "Regularized Constrained Total Least Squares Image Restoration”, IEEE Trans. Image Processing, vol. 4, pp.10961107. Aug. 1995

[3]. W. F Chen, M. Chen, and J. Zhou, “Adaptively Regularized Constrained Total Least-Squares Image Restoration", IEEE Trans. Image Processing, vol.9, pp. 588-596. April 2000.

[4]. P. L. Combettes and H. J. Trussell, "Methods for digital restoration of signals degraded by a stochastic impulse response”, IEEE Trans. Acoust., Speech, Signal Processing, vol. 37, no.3, pp. 393-401, March 1989.

[5]. X. C. Gan, Alan, W. L. Liew and H. Yan, "An Efficient onstrained Total Least Squares Algorithm for Image Restoration Using Dependent Random Variables Set”, IEEE Trans. Signal Processing, submitted.

[6]. P. Lemmerling, S. Van Huffel, B. De Moor, "Structured total least squares problems: formulations, algorithms and applications", in Recent Advances in Total Least Squares Techniques and Errors-in-Variables modeling SIAM, Philadelphia, 1997, pp. 215-223. Acoust., Speech, Signal Processing, vol.37, no. 3, pp.393-401, Mar.1989.

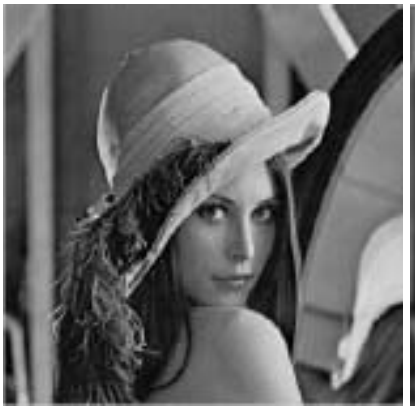

a)

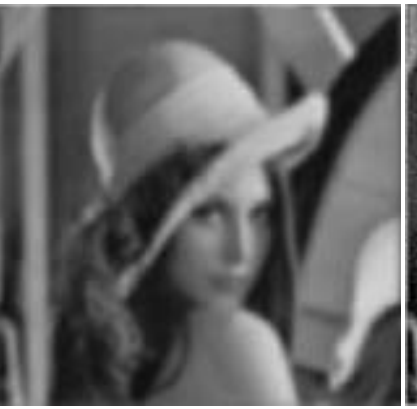

b)

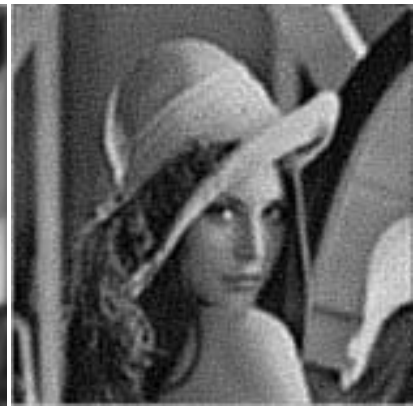

c)

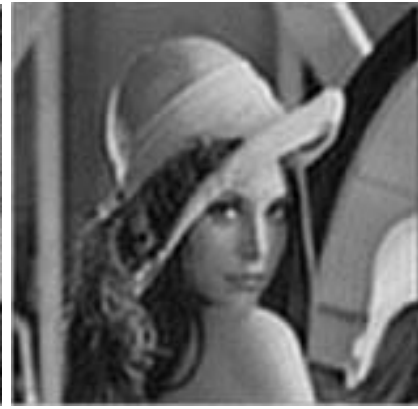

d)

Fig. 1 Objective elevation of algorithm a) Origin image; b) Degraded image; c) Restored image with Mesarovic algorithm; e) Restored image with our method 